\title{
Nachtlärminduzierte Schlafstörungen und Herz-Kreislauf-Risiko
}

\section{Nighttime Noise-induced Sleep Disorders and Cardiovascular Risk}

\section{다)(ㅇ) ()}

Autoren

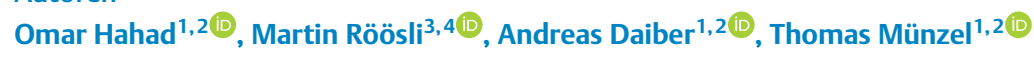

Institute

1 Zentrum für Kardiologie, Kardiologie I, Universitätsmedizin der Johannes Gutenberg-Universität Mainz, Mainz, Deutschland

2 Deutsches Zentrum für Herz-Kreislauf-Forschung (DZHK), Standort Rhein-Main, Mainz, Deutschland

3 Schweizerisches Tropen- und Public Health-Institut, Basel, Schweiz

4 Universität Basel, Basel, Schweiz

\section{Schlüsselwörter}

Schlafstörungen, Herz-Kreislauf-Risiko, nächtlicher Lärm

Key words

sleep disorders, cardiovascular risk, nighttime noise

\section{Bibliografie}

Aktuel Kardiol 2021; 10: 521-525

DOI 10.1055/a-1545-0604

ISSN 2193-5203

(C) 2021. The Author(s).

This is an open access article published by Thieme under the terms of the Creative Commons Attribution-NonDerivative-NonCommercial-License, permitting copying and reproduction so long as the original work is given appropriate credit. Contents may not be used for commercial purposes, or adapted, remixed, transformed or built upon. (https://creativecommons.org/licenses/by-nc-nd/4.0/).

Georg Thieme Verlag KG, Rüdigerstraße 14,

70469 Stuttgart, Germany

Korrespondenzadresse

Dr. Omar Hahad

Zentrum für Kardiologie, Kardiologie I

Universitätsmedizin der Johannes Gutenberg-Universität

Mainz

Langenbeckstr. 1

55131 Mainz, Deutschland

omar.hahad@unimedizin-mainz.de

\section{ZUSAMMENFASSUNG}

Beeinträchtigungen des Schlafes im Sinne von Insomnien, d. h. Ein- und Durchschlafschwierigkeiten, frühmorgendliches Erwachen und ein chronisch unerholsamer Schlaf, sind weit verbreitet in der Bevölkerung und gehen mit einer Vielzahl von körperlichen und psychischen Gesundheitsstörungen einher. Die umweltbedingten Ursachen für Schlafstörungen umfassen eine ganze Reihe von Faktoren, wobei nächtlicher Lärm als eine wichtige Ursache gilt. Aktuelle Daten der Europäischen Umweltagentur zeigen, dass Umgebungslärm (Straßenverkehrs-, Schienen-, Flug- und Industrielärm) für starke Schlafstörungen bei 6,5 Millionen Personen in Europa sorgt. Neue epidemiologische und mechanistische Feldstudien zeigen, dass vor allem nächtlicher Verkehrslärm unter anderem zu einer eingeschränkten Gefäßfunktion, thromboinflammatorischen Veränderungen, einem Anstieg von Stresshormonen und einem erhöhten Blutdruck führen kann, die bedeutsame Risikofaktoren für Herz-Kreislauf-Erkrankungen darstellen. Gemäß der Europäischen Umweltagentur führt der Umgebungslärm jährlich zu 48000 zusätzlichen Fällen von ischämischen Herzerkrankungen sowie 12000 vorzeitigen Todesfällen.

\section{ABSTRACT}

Impairment of sleep in the sense of insomnias, i.e. difficulties falling asleep and staying asleep, early morning awakening and chronical unrestful sleep is widespread in the population and are associated with a variety of physical and mental health disorders. The environmental causes of insomnia include a number of factors, with nighttime noise being an important cause. Recent data from the European Environment Agency shows that environmental noise (road traffic, railway, aircraft and industrial noise) causes severe sleep disorders among 6.5 million people in Europe. New epidemiological and mechanistic field studies show that in particular night traffic noise can lead, among other things, to impaired vascular function, thrombo-inflammatory changes, an increase in stress hormones and increased blood pressure, representing significant risk factors for cardiovascular diseases. According to the European Environment Agency, environmental noise causes 48000 additional cases of ischemic heart disease and 12000 premature deaths each year. 


\section{Glossar}

DALY

EEA

FMD

WHO
Disability-adjusted Life Year

Europäische Umweltagentur

flussvermittelte Vasodilatation

Weltgesundheitsorganisation

\section{WAS IST WICHTIG?}

Umgebungslärm ist eine der wichtigsten Ursachen für umweltbedingte Schlafstörungen. Einerseits verursacht Nachtlärm Einschlafschwierigkeiten sowie Aufwachreaktionen. Letzteres tritt insbesondere am Morgen auf, wenn der Schlafdruck geringer ist. Andererseits aktiviert Tageslärm das Stresssystem, was bis in die Schlafphasen hinein andauern kann und damit die Regenerationswirkung des Schlafes beeinträchtigt. Verkehrslärm ist somit ein bedeutsamer Risikofaktor für Herz-Kreislauf-Erkrankungen, wobei lärmbedingte Schlafstörungen einen relevanten Mechanismus bei der Vermittlung der negativen Herz-Kreislauf-Auswirkungen wie eine eingeschränkte Gefäßfunktion und Bluthochdruck darstellen.

\section{Lärmbedingte Schlafstörungen als bedeutsame Determinante der öffentlichen Gesundheit}

Menschen verbringen etwa ein Drittel ihrer Lebenszeit mit Schlaf, wobei dieser einen überlebensnotwendigen Prozess darstellt und eine fundamentale Rolle im Hinblick auf biologische und psychische Regenerationsprozesse spielt. Nächtliche Schlafstörungen im Sinne einer Insomnie, d.h. Ein- und Durchschlafschwierigkeiten, frühmorgendliches Erwachen und ein chronisch unerholsamer Schlaf, sind weit verbreitete Erscheinungen in der Bevölkerung und gehen mit psychosozialen Beeinträchtigungen wie Tagesmüdigkeit, reduzierter Konzentrationsfähigkeit, Stress, schlechterem allgemeinen Gesundheitszustand und mangelndem psychischem Wohlbefinden sowie der Einschränkung von sozialen Kontakten einher. Die gesundheitsökonomischen und volkswirtschaftlichen Kosten von Schlafstörungen sind enorm, vor allem bedingt durch Krankheitsfehltage, Leistungsabfall und Produktivitätsverlust. Schätzungen epidemiologischer Studien zufolge schwankt die weltweite Prävalenz von Schlafstörungen im Sinne einer Insomnie zwischen 10-30\% [1].

In der Studie zur Gesundheit von Erwachsenen ( $n=8152$, Alter 18-79 Jahre) in Deutschland (DEGS1) berichtete etwa ein Drittel der Befragten über potenziell klinisch relevante Ein- oder Durchschlafstörungen und etwa ein Fünftel zusätzlich über eine schlechte Schlafqualität, wobei insgesamt eine Prävalenz von 5,7\% für ein Insomniesyndrom ermittelt wurde [1]. In der aktuelleren DAK-Studie bei Erwerbstätigen von 2017 ( $n=5207$, Alter 18-65 Jahre) wurde für den Vergleich mit den Ergebnissen aus dem Jahre 2010 gezeigt, dass die Prävalenz der Menschen im Alter von 35-65 Jahren, die unter Ein- oder Durchschlafstörungen litten, von 47,5\% auf 78,9\% zugenommen hat, wobei lediglich 21,1\% der Befragten berichteten, keine Schlafprobleme zu haben [2]. Dabei ist wichtig

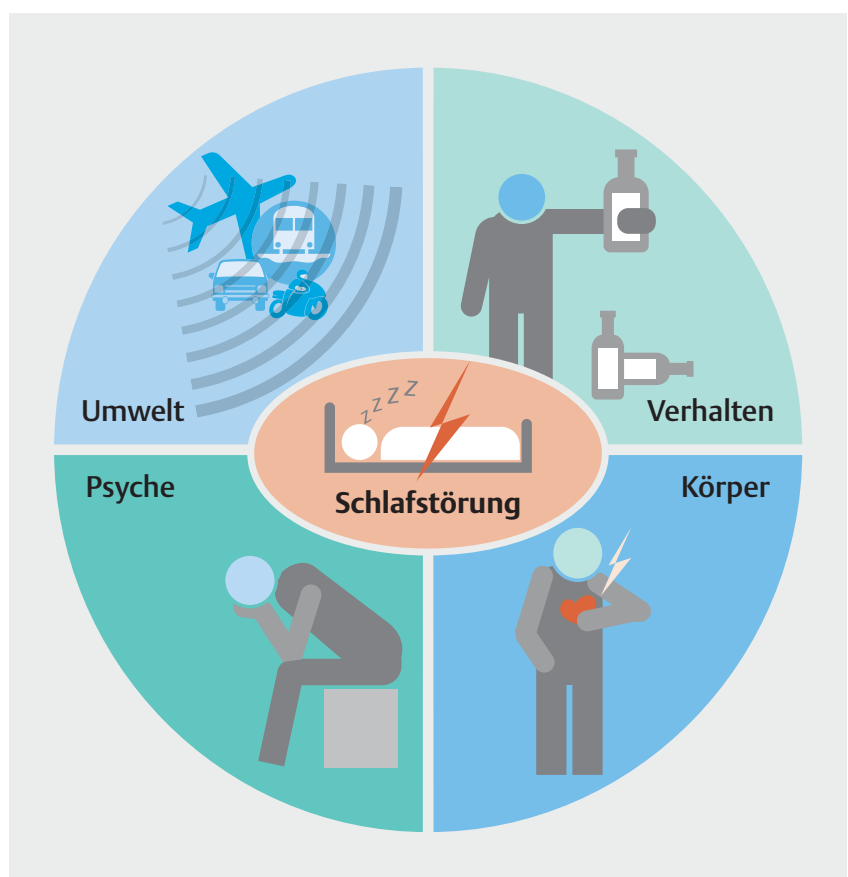

Abb. 1 Ursachenbereiche für Schlafstörungen. Es werden körperliche (z. B. schmerzhafte Erkrankungen, Inkontinenz), psychische (z. B. Stress, psychische Störungen), verhaltens- (z. B. Rauchen, Alkohol) sowie umweltbedingte (z. B. Lärm, Licht) Ursachen für Schlafstörungen unterschieden.

zu erwähnen, dass sowohl ein verkürzter Schlaf, aber auch eine erhöhte Schlafdauer (Hypersomnie), mit einem erhöhten Risiko für verschiedene psychische und körperliche Erkrankungen sowie mit einer erhöhten Sterblichkeit einhergeht (7-9 Stunden gelten als normale Schlafdauer für gesunde Erwachsene). Ein verkürzter Schlaf ist mit einer Reihe von Risikofaktoren, wie Bluthochdruck, Übergewicht und Adipositas sowie metabolisches Syndrom, assoziiert und kann darüber vermittelt das Risiko für Herz-Kreislauf-Erkrankungen wie koronare Herzerkrankung und Schlaganfall erhöhen. Dagegen ist der Zusammenhang zwischen einer erhöhten Schlafdauer und einem erhöhten Krankheitsrisiko vermutlich vor allem dadurch bedingt, dass Menschen mit einer höheren Schlafdauer auch an anderen bestehenden Erkrankungen leiden. Grundsätzlich sollte festgehalten werden, dass der Gesundheits- bzw. Krankheitszustand und das Schlafverhalten in einem bidirektionalen Verhältnis stehen - demnach kann das Schlafverhalten den Gesundheits- bzw. Krankheitszustand beeinflussen und andersherum kann der Gesundheits- bzw. Krankheitszustand das Schlafverhalten beeinflussen.

Trotz der bereits gut untersuchten Zusammenhänge zwischen Schlaf und Gesundheit wird die Relevanz des Schlafes als Determinante der öffentlichen Gesundheit noch immer unterschätzt. Als wesentlicher Einflussfaktor für das Auftreten von Schlafstörungen gelten neben den bereits genannten Beeinträchtigungen der körperlichen sowie psychischen Gesundheit und Verhaltensaspekte vor allem Umwelteinflüsse ( $\triangleright$ Abb. 1). Die umweltbedingten Ursachen für Schlafstörungen umfassen eine ganze Reihe von Faktoren wie Lärm, Licht, Temperatur/Wetter, Luftqualität und Feuchtigkeit. 
Vor allem der Umgebungslärm, der zu den größten Umweltproblemen unserer Zeit gehört, gilt hier als eine bedeutsame umweltbedingte Ursache. In einem Bericht der Weltgesundheitsorganisation (WHO) zur Krankheitslast aufgrund von Umgebungslärm wird davon ausgegangen, dass Umgebungslärm, vor allem bedingt durch Transport und Verkehr, für den Verlust von nahezu 1,7 Millionen gesunden Lebensjahren (DALYs) pro Jahr in den Mitgliedstaaten der Europäischen Union sowie weiteren westeuropäischen Staaten verantwortlich ist [3]. Dabei sorgen lärmbedingte Schlafstörungen sowie Belästigungsreaktionen mit 903000 bzw. 654000 Jahren für den größten Anteil an verlorenen gesunden Lebensjahren. Aktuelle Daten der Europäischen Umweltagentur (EEA) zeigen, dass Umgebungslärm (Straßenverkehrs-, Schienen-, Flug- und Industrielärm) für starke Lärmbelästigung bei 22 Millionen Personen sowie für starke Schlafstörungen bei 6,5 Millionen Personen in Europa sorgt, was jährlich zu den 48000 zusätzlichen Fällen von ischämischen Herzerkrankungen sowie 12000 vorzeitigen Todesfällen wegen Lärm beiträgt [4]. In dieser Übersicht wird die Relevanz von Umgebungslärm als Ursache für Schlafstörung erläutert und beschrieben, wie lärmbedingte Schlafstörungen das Risiko für Herz-Kreislauf-Erkrankungen erhöhen können.

\section{KURZGEFASST}

Schlafstörungen (Insomnien) sind in der Bevölkerung weit verbreitet und gehen mit einer Vielzahl von körperlichen und psychischen Gesundheitsstörungen einher. Vor allem Umgebungslärm, der als der am stärksten störende Umweltfaktor gilt, ist eine bedeutsame Ursache für umweltbedingte Schlafstörungen, wobei lärmbedingte Schlafstörungen das Herz-Kreislauf-Risiko erhöhen können.

\section{Lärmbedingte Schlafstörungen}

Die WHO hat 2009 einen Bericht veröffentlicht, der sich schwerpunktmäßig mit Nachtlärm befasst. Laut diesem Bericht „gibt es Belege aus Tier- und Menschenstudien, die die Hypothese unterstützen, dass Nachtlärm stärker als Tageslärm mit Herz-KreislaufEreignissen in Verbindung steht“. Dies unterstreicht den Bedarf an weiteren epidemiologischen Studien zu diesem Thema [5]. Seit 2009 wurden folglich in einer Reihe von Feldstudien, experimentellen Studien und epidemiologischen Studien die Herz-KreislaufAuswirkungen von Nachtlärm untersucht, die zeigen, dass dieses Zeitfenster besonders entscheidend sein könnte, da die Lärmbelästigung in der Nacht den Schlaf stört und darüber verschiedene Herz-Kreislauf-Risikofaktoren begünstigt [6]. Dies wird auch durch Studien belegt, die zeigen, dass Probanden, die ihre Schlafzimmerfenster zur dem Straßenverkehr abgewandten Seite haben, weniger stark durch den Straßenverkehrslärm gesundheitlich betroffen sind.

So ergab eine aktuelle Metaanalyse, die im Auftrag der WHO durchgeführt wurde, dass Verkehrslärmexposition das Risiko für subjektiv wahrgenommene Schlafstörungen erhöhen kann, wobei Fluglärmexposition mit einem fast 2-fach erhöhten Risiko für starke Schlafstörungen pro Zunahme von 10 Dezibel des Nachtlärmpegels ( $\left.L_{\text {night }}\right)$ einherging [7]. Zudem zeigte hier die Metaanalyse polysomnografischer Daten, dass ein Anstieg von 10 Dezibel des maximalen Nachtinnenraumgeräuschpegels durch Fluglärm mit einer um 35\% erhöhten Wahrscheinlichkeit nächtlichen Erwachens verbunden ist (d.h. für einen Wechsel von einer Tiefschlafphase bzw. Mitteltiefschlafphase zum Wachzustand oder Leichtschlafphase). Eine weitere aktuelle Studie aus Frankreich konnte zeigen, dass sowohl die Zahl nächtlicher Fluglärmereignisse als auch die Fluglärmpegel einen negativen Einfluss auf objektive ermittelte Parameter der Schlafqualität ausüben [8]. Darüber hinaus konnten dieselben Forscher auch den negativen Einfluss nächtlicher Fluglärmexposition hinsichtlich der subjektiv bewerteten Schlafqualität demonstrieren anhand einer kürzeren Gesamtschlafzeit sowie erhöhter Müdigkeit am nächsten Morgen [9]. In Übereinstimmung mit diesem Ergebnis zeigen mehrere Beobachtungsstudien zu Verkehrslärm, dass Lärmexposition den stärksten Effekt auf die subjektiv wahrgenommene Schlafqualität am Morgen hat. In einer norwegischen Studie ( $n=13019)$ [10] sowie einer Studie aus der Schweiz ( $n=1375)$ [11] war die nächtliche Verkehrslärmexposition vorwiegend mit einem frühen selbstberichteten Aufwachen verbunden, während Zusammenhänge mit anderen Schlafqualitätsparametern, wie zum Beispiel Durch- oder Einschlafprobleme, weniger ausgeprägt waren.

\section{KURZGEFASST}

Nachtlärm führt zu objektiv messbaren Aufwachreaktionen und subjektiv reduzierter Schlafqualität. Besonders ausgeprägt ist der Lärmeffekt am Morgen, wenn der Schlafdruck weniger hoch ist.

\section{Nächtlicher Lärm und Herz-Kreislauf-Risiko}

Wenige mechanistische Feldstudien haben sich mit den HerzKreislauf-Auswirkungen des nächtlichen Verkehrslärms beschäftigt. In 4 Feldstudien haben wir die Effekte von nächtlichem Flugund Schienenverkehrslärm auf die Gefäßfunktion - genauer Endothelfunktion, bestimmt als flussvermittelte Vasodilatation (FMD) der A. brachialis, die als ein wichtiger, früher Risikomarker für die Entwicklung von Herz-Kreislauf-Erkrankungen gilt - bei gesunden Probanden und bei Patienten mit bestätigter koronarer Herzkrankheit untersucht $[12,13,14,15]$. In diesen Studien verursachte der simulierte nächtliche Verkehrslärm eine deutliche Verschlechterung der Schlafqualität, eine Verschlechterung der Gefäßfunktion und teilweise einen Anstieg von Stresshormonen, einen erhöhten Blutdruck, eine erhöhte Gefäßsteifigkeit, thromboinflammatorische Veränderungen sowie eine verschlechterte diastolische Funktion des Herzens ( $\mathbf{A} \mathbf{b} \mathbf{b}$. 2). Die negativen Effekte von nächtlichem Lärm auf die Endothelfunktion waren bei Probanden mit koronarer Herzerkrankung ausgeprägter. In kleineren Teilgruppen mit gesunden Probanden konnte die Einnahme des Antioxidans Vitamin C die FMD signifikant verbessern, was vermuten lässt, dass Nachtlärm eine endotheliale Dysfunktion hauptsächlich durch die erhöhte vaskuläre Formation von reaktiven Sauerstoffspezies induziert, wodurch die vaskuläre Verfügbarkeit von Stickstoffmonoxid verringert wird. Zudem sind Schlafstörungen per se mit einer endothelialen Dysfunktion bei gesunden Proban- 


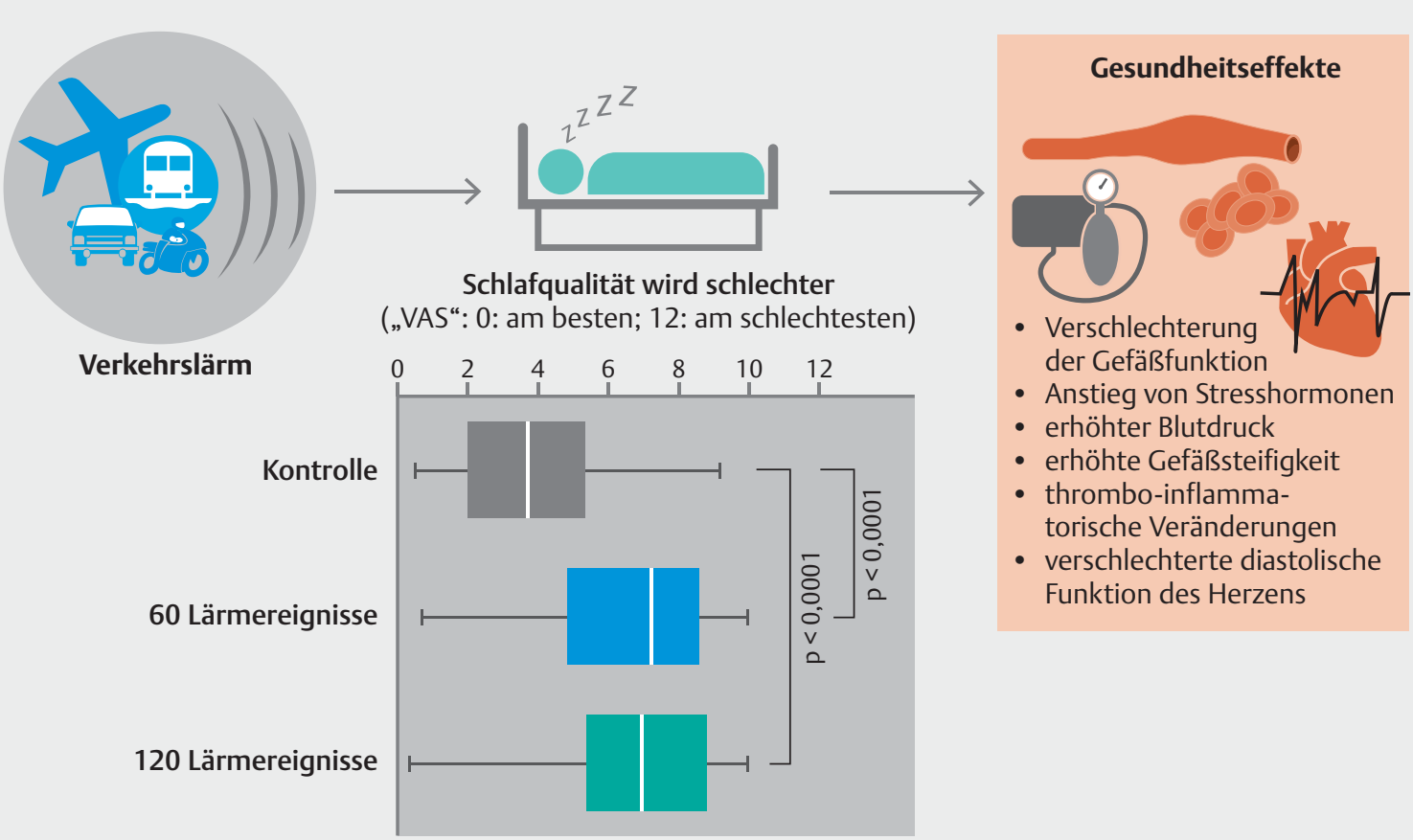

- Abb. 2 Zusammenfassende Darstellung der Ergebnisse unserer Feldstudien zum Einfluss von nächtlichem Verkehrslärm auf das Herz-KreislaufSystem $[12,13,14,15]$. Der Graph zur Schlafqualität wurde aus Rohdaten der FluG-prime-Studie generiert. Gezeigt sind die Daten als Median mit $\mathrm{min} / \mathrm{max}$ Fehlerbalken [15]. VAS: visuelle Analogskala

den assoziiert. Weiterhin konnte in einer der Feldstudien demonstriert werden, dass die lärmbedingte Verschlechterung der Gefäßfunktion besonders dann ausgeprägt war, wenn die Probanden zunächst einem Szenario exponiert wurden, das 30 Lärmereignisse und darauffolgend 60 Lärmereignisse beinhaltete, sodass auf Gefäßebene eher eine lärmbedingte Sensibilisierung als eine Habituation zu beobachten war (Priming-Effekt) [12, 13, 14, 15].

\section{KURZGEFASST}

Feldstudien zeigen, dass simulierter nächtlicher Verkehrslärm zu einer Verschlechterung der Schlafqualität, einer eingeschränkten Gefäßfunktion, einem Anstieg von Stresshormonen, einem erhöhten Blutdruck, einer erhöhten Gefäßsteifigkeit, thromboinflammatorischen Veränderungen sowie einer verschlechterten diastolischen Funktion des Herzens führen kann, die insgesamt relevante Mechanismen darstellen, wie Nachtlärm das Herz-Kreislauf-Risiko erhöhen kann.

\section{Schlussfolgerungen}

Schlafstörungen stellen einen relevanten Mechanismus bei der Vermittlung der negativen Herz-Kreislauf-Auswirkungen von nächtlichem Lärm dar. Nächtlicher Verkehrslärm steht mit reduzierter Schlafqualität, Durchschlafschwierigkeiten und Veränderungen der Schlafphasen in Zusammenhang. Die Evidenz aus tier- experimentellen sowie humanen epidemiologischen und Feldstudien zeigt, dass Nachtlärm im Vergleich zum Tageslärm mit stärkeren negativen Effekten auf das Herz-Kreislauf-System in Verbindung steht. Im Vergleich zum Tageslärm führt Nachtlärm zu einer stärkeren hormonellen Stressreaktion, einer erhöhten Konzentration von reaktiven Sauerstoffspezies, einer ausgeprägteren Störung der Gefäßfunktion und -steifigkeit, Bluthochdruck und folglich laut epidemiologischen Studien zu einer höheren Inzidenz von Herz-Kreislauf-Erkrankungen. Die stärkere Auswirkung des Nachtlärms auf die Gesundheit bedeutet jedoch nicht, dass Tageslärm unbedenklich ist. Tageslärm kann mit psychischen (Ärgerreaktionen und Depressionen) und physiologischen Stressreaktionen einhergehen, die bis in die Schlafphasen hinein andauern können und die Regenerationswirkung des Schlafes beeinträchtigen. Daher sollten präventive Maßnahmen ihren Schwerpunkt sowohl auf den Tages- als auch auf dem Nachtlärm legen.

\section{Fazit}

Nächtlicher Verkehrslärm ist ein bedeutsamer Risikofaktor für Herz-Kreislauf-Erkrankungen. Dabei stellen lärmbedingte Schlafstörungen einen relevanten Mechanismus bei der Vermittlung der negativen Herz-Kreislauf-Auswirkungen dar. Zudem können psychische und physiologische Stressreaktionen, die mit Tageslärm assoziiert sind, bis in die Schlafphasen hinein andauern und die Regenerationswirkung des Schlafes beeinträchtigen. 
Interessenkonflikt

Die Autorinnen/Autoren geben an, dass kein Interessenkonflikt besteht.

Literatur

[1] Schlack R, Hapke U, Maske U et al. Häufigkeit und Verteilung von Schlafproblemen und Insomnie in der deutschen Erwachsenenbevölkerung. Bundesgesundheitsbl 2013; 56: 740-748. doi:10.1007/s00103-013-168 9-2

[2] Losch D, Klingelhöfer D, Groneberg DA. Schlafstörungen und Arbeitsunfähigkeit in Deutschland. Zbl Arbeitsmed 2017; 67: 342-344. doi:10.100 7/s40664-017-0214-0

[3] World Health Organization. Burden of disease from environmental noise. Quantification of healthy life years lost in Europe. 2011. Im Internet (Stand: 03.10.2021): https://www.euro.who.int/_data/assets/pdf_file/ 0008/136466/e94888.pdf

[4] European Environment Agency. Environmental noise in Europe - 2020. 2020. Im Internet (Stand: 03.10.2021): https://www.eea.europa.eu/publications/environmental-noise-in-europe

[5] World Health Organization. Night noise guidelines for Europe. 2009. Im Internet (Stand: 03.10.2021): https://www.euro.who.int/_data/assets/ pdf_file/0017/43316/E92845.pdf

[6] Munzel T, Kroller-Schon S, Oelze M et al. Adverse Cardiovascular Effects of Traffic Noise with a Focus on Nighttime Noise and the New WHO Noise Guidelines. Annu Rev Public Health 2020; 41: 309-328. doi:10.1146/ann urev-publhealth-081519-062400

[7] Basner M, McGuire S. WHO Environmental Noise Guidelines for the European Region: A Systematic Review on Environmental Noise and Effects on Sleep. Int J Environ Res Public Health 2018; 15: 519. doi:10.3390/ijerp h15030519

[8] Nassur AM, Leger D, Lefevre $M$ et al. The impact of aircraft noise exposure on objective parameters of sleep quality: results of the DEBATS study in France. Sleep Med 2019; 54: 70-77. doi:10.1016/j.sleep.2018.1 0.013

[9] Nassur AM, Lefevre M, Laumon B et al. Aircraft Noise Exposure and Subjective Sleep Quality: The Results of the DEBATS Study in France. Behav Sleep Med 2019; 17: 502-513. doi:10.1080/15402002.2017.1409224

[10] Evandt J, Oftedal B, Hjertager Krog N et al. A Population-Based Study on Nighttime Road Traffic Noise and Insomnia. Sleep 2017. doi:10.1093/sle ep/zsw055

[11] Frei P, Mohler E, Roosli M. Effect of nocturnal road traffic noise exposure and annoyance on objective and subjective sleep quality. Int J Hyg Environ Health 2014; 217: 188-195. doi:10.1016/j.ijheh.2013.04.003

[12] Schmidt FP, Basner M, Kroger $G$ et al. Effect of nighttime aircraft noise exposure on endothelial function and stress hormone release in healthy adults. Eur Heart J 2013; 34: 3508-3514a. doi:10.1093/eurheartj/eht269

[13] Schmidt F, Kolle K, Kreuder K et al. Nighttime aircraft noise impairs endothelial function and increases blood pressure in patients with or at high risk for coronary artery disease. Clin Res Cardiol 2015; 104: 23-30. doi:1 0.1007/s00392-014-0751-x

[14] Herzog J, Schmidt FP, Hahad O et al. Acute exposure to nocturnal train noise induces endothelial dysfunction and pro-thromboinflammatory changes of the plasma proteome in healthy subjects. Basic Res Cardiol 2019; 114: 46. doi:10.1007/s00395-019-0753-y

[15] Schmidt FP, Herzog J, Schnorbus B et al. The impact of aircraft noise on vascular and cardiac function in relation to noise event number: a randomized trial. Cardiovasc Res 2021; 117: 1382-1390. doi:10.1093/cvr/c vaa204 